\title{
Diffusivity of Mixtures in Warm Dense Matter Regime
}

T. Haxhimali, R. E. Rudd

June 17, 2013

Computational Challenges in Warm Dense Matter Santa Monica, CA, United States May 21, 2012 through May 25, 2012 
This document was prepared as an account of work sponsored by an agency of the United States government. Neither the United States government nor Lawrence Livermore National Security, LLC, nor any of their employees makes any warranty, expressed or implied, or assumes any legal liability or responsibility for the accuracy, completeness, or usefulness of any information, apparatus, product, or process disclosed, or represents that its use would not infringe privately owned rights. Reference herein to any specific commercial product, process, or service by trade name, trademark, manufacturer, or otherwise does not necessarily constitute or imply its endorsement, recommendation, or favoring by the United States government or Lawrence Livermore National Security, LLC. The views and opinions of authors expressed herein do not necessarily state or reflect those of the United States government or Lawrence Livermore National Security, LLC, and shall not be used for advertising or product endorsement purposes. 


\title{
Diffusivity of Mixtures in Warm Dense Matter Regime
}

\author{
Tomorr Haxhimali and Robert E. Rudd
}

\begin{abstract}
Modeling of ionic diffusion in warm dense plasma mixtures has been of longstanding interest in astrophysics and in Inertial Confinement Fusion. Here we review traditional approaches to calculating plasma diffusion using kinetic theory. We also review earlier classical molecular dynamics (MD) results. We discuss some new results from MD for self and mutual diffusion in a mixture of deuterium and argon at warm dense matter regime. We make use of Yukawa interionic potentials as an effective potential that accounts for the screening effects of the electrons to the ions. We further provide a general description of the Green-Kubo technique to extract the diffusivity of a multicomponent mixture. The description is very general and it can be extended to plasmas.
\end{abstract}

Tomorr Haxhimali

Lawrence Livermore National Laboratory,

Livermore, California, 94550, USA, e-mail: haxhimalilellnl.gov

Robert E. Rudd

Lawrence Livermore National Laboratory,

Livermore, California, 94550, USA, e-mail: robert.ruddellnl.gov 


\section{Introduction}

Species diffusion is the process in which a net species flux arises in the center of mass frame in response to gradients in the concentration, pressure, or temperature.[1] The species may be atoms or molecules or even larger particles, depending on the system, but for the purpose of this article the species will be different kinds of ions. Species diffusion is often in response to concentration gradients. The species current lowers the free energy by increasing the entropy of mixing, so species diffusion is necessarily a non-equilibrium process. Temperature gradients and pressure gradients may also give rise to gradients in the chemical potential that drive diffusion. These are special cases of species diffusion called thermodiffusion and barodiffusion, and they can be important in plasmas [2, 3, 4]. This article focuses primarily on species diffusion driven by concentration gradients, and for simplicity we will refer to it as diffusion.

Diffusion is an important component of mixing. Mixing is a combination of stirring and diffusion, in which stirring is hydrodynamic flow that redistributes initially separated species, and diffusion causes them to mix irreversibly at the atomic level. At high Reynolds numbers vortices can develop and cause stirring on many length scales, and diffusion operates on this complex flow pattern. At low Reynolds numbers, fluid flow is governed by the Stokes equation, so it is reversible. Two initially separated components can be mixed by stirring, and then unmixed by stirring in the reverse direction. Experiments that demonstrate this effect are quite striking [5]. Only a very small amount of mixing persists due to the irreversible diffusion.

Within plasma physics there are well known examples where diffusion is understood to play an important role or where it has been proposed to be important. It is key to the purification of white dwarf atmospheres through the sedimentation of heavy elements.[6] A similar phenomenon is thought to occur in neutron stars. Diffusion can also have an effect in hot spot ignition for inertial confinement fusion (ICF), since it is a mechanism for ablator materials to degrade the fuel.[7, 8] Also, other systems exhibit diffusion of particles interacting through screened Coulomb interactions, such as dust particles in plasmas and colloidal particles suspended in electrolyte [9, 10, 11, 12]. Diffusion in these systems spans a large range of regimes. In ICF, the fuel starts cold and at solid density $\sim 1 \mathrm{~g} / \mathrm{cm}^{3}$, is rapidly compressed to warm dense matter conditions, and ultimately while burning should be at temperatures $\sim 1 \mathrm{keV}$ and densities of $\sim 1000 \mathrm{~g} / \mathrm{cm}^{3}$. [7] A white dwarf is at a temperature of $\sim 20$ to $1000 \mathrm{eV}$ and a broad range of densities. The white dwarf is a weakly coupled plasma, whereas the ICF fuel goes from strongly coupled to weakly coupled. Understanding diffusion across this range of conditions is a challenge.

There are several classic pictures of diffusion. In a simplified picture from the kinetic theory of gases, atoms move ballistically on the average of some length $\lambda$, the mean free path, before scattering. After scattering, they move ballistically in a different direction. If there is no concentration gradient, there will be just as many atoms coming from one direction as from the opposite direction, so there is no net flux. If there is a concentration gradient, more atoms will come from the region with higher concentration, so the flux will be proportional to the concentration gradient. 
If the pressure and temperature are uniform, the mass flux $\mathbf{J}$ is related to the gradient in the mass fraction of one species, $c(\mathbf{r})$, according to:[13]

$$
\mathbf{J}=-\rho D_{12} \nabla c(\mathbf{r})
$$

where $\rho$ is the mass density. The coefficient of proportionality, $D_{12}$, is the mutual diffusivity or interdiffusivity. In the simplified ideal gas picture, it is related to the mean free path and mean thermal velocity, $v_{t h}$, by the relation:

$$
D_{12}=\frac{1}{3} \lambda v_{t h}
$$

Since mass is conserved, the mass flux obeys a continuity equation:

$$
\rho\left(\partial_{t} c+\mathbf{v} \cdot \nabla c(\mathbf{r})\right)=-\nabla \cdot \mathbf{J}
$$

Together eqs. (1) and (3) give rise to the equation:

$$
\frac{\partial c}{\partial t}=D_{12} \nabla^{2} c(\mathbf{r})
$$

where we have taken $\mathbf{v}=0$. Equation (1) is known as Fick's first law of diffusion, and eq. (4) is known as Fick's second law of diffusion. In the simplified kinetic theory, both the mean free path $\lambda$ and the thermal velocity $v_{t h}$ are positive, so the mutual diffusivity is positive. The mass flux acts to decrease the gradients, and the diffusivity increases with increasing thermal velocity and mean free path.

Another familiar case is the diffusion of an interstitial atom in a crystal lattice. The interstitial is an extra atom sitting in a site between the regularly arrayed atoms of the crystal. Interstitials can diffuse, hopping from one interstitial site to another, but there is an energy barrier that must be overcome to hop from site to site. The energy to overcome the barrier is provided by thermal fluctuations. So the atom rattles around in the energy well of one interstitial site until a sufficiently large thermal fluctuation pushes it over the barrier. A similar phenomenon occurs in liquids in which atoms are trapped in the cage of their neighbors for some period of time until a thermal fluctuation allows them to escape the cage. Diffusion of the interstitial atom in a crystal or a caged atom in a liquid is punctuated by hops, just as diffusion in the ideal gas is punctuated by scattering events. After many hops, the distribution function describing the probability of finding the atom a distance $r$ from its starting point is a Gaussian in $r$ whose width increases like the square-root of time, characteristic of diffusion.

These two kinds of diffusive phenomena also occur in plasmas. Weakly coupled plasmas exhibit diffusion like the ideal gas, while strongly coupled plasmas diffuse like the liquid. In plasmas the scattering is due to the Coulomb repulsion between like-charged ions, screened by the electrons. The scattering cross-section that determines the mean free path involves an integral that diverges logarithmically at both short and long range for the pure Coulomb interaction. It is cutoff at long ranges by the Debye length when screening is included, and at short distances by the classical 
turning point. The resulting kinetic theory making use of Chapman-Enskog theory provides a good description of diffusion in weakly coupled plasmas. Chapman and Cowling derived analytic expressions for diffusion in Coulomb systems, [14] and Paquette extended this work to statically screened ions.[15]

In the strongly coupled regime, cage effects become important, invalidating the binary collision model upon which the Chapman-Cowling and Paquette models are based. In this regime, molecular dynamics (MD) has been used to capture the manybody effects governing diffusion.[16] There has been a recent finding that effective pair potentials provided a means of extended the realm of validity of a diffusion model based on binary collisions.[17] The MD simulations valid at strong coupling, become more challenging when the coupling is weak.

We now give a brief review of the parameters of a mixed plasma. We assume a mixture of the ion species $\alpha=1,2, \ldots$ with mass and charge $A_{\alpha}$ and $Z_{\alpha}$, respectively. The total number density of ions is given by $n=\sum_{\alpha} n_{\alpha}$, where $n_{\alpha}$ is the number density of the ions of species $\alpha$. The Wigner-Seitz radius of the system is given by: $a_{W S}=\left(\frac{4}{3} \pi n\right)^{-1 / 3}$. From the charge neutrality of the mixture the number density of the electrons is:

$$
n_{e}=\langle Z\rangle n
$$

where $\langle Z\rangle$ is the average ionization of the mixture. We follow the general definition for the angular brackets $\langle\cdots\rangle$ denoting a number weighted average of a parameter $\Xi$ in a mixture of the type $\langle\Xi\rangle=\sum_{\alpha} \Xi_{\alpha} X_{\alpha}$ where $X_{\alpha}=n_{\alpha} / n$ is the mole fraction of species $\alpha$. The state of the free electrons is determined by the electron number density $n_{e}$ and temperature $T$. It is convenient to introduce the dimensionless density parameter $r_{s}=a_{e} / a_{0}$, where $a_{e}=\left(\frac{4}{3} \pi n_{e}\right)^{-1 / 3}$ and $a_{0}$ is the Bohr radius. The degeneracy of the electrons in the system is determined by the parameter $\Theta \equiv k_{B} T / E_{F}$, where $E_{F} \equiv \frac{\hbar^{2}}{2 m_{e}}\left(3 \pi^{2} n_{e}\right)^{2 / 3}$ is the Fermi energy and $m_{e}$ the mass of the electron.

The strength of the ion-ion Coulomb interaction in species $i$ is characterized by the coupling parameter:

$$
\Gamma_{i}=\frac{\left(Z_{i} e\right)^{2}}{4 \pi \varepsilon_{0} a_{i} k_{B} T}=Z_{i}^{5 / 3}\langle Z\rangle^{1 / 3} \Gamma
$$

where $a_{i}=\frac{Z_{i}^{1 / 3}}{\langle Z\rangle^{1 / 3}} a_{W S}$ is the ion sphere radius introduced by Salpeter [18], and $\Gamma \equiv$ $e^{2} /\left(4 \pi \varepsilon_{0} a_{W S} k_{B} T\right)$ is the electron-electron coupling parameter. In a multicomponent mixed plasma it is useful to introduce an effective coupling [19, 20, 21, 22, 23]:

$$
\Gamma_{e f f}=\sum_{i} X_{i} \Gamma_{i}=\left\langle Z^{5 / 3}\right\rangle\langle Z\rangle^{1 / 3} \Gamma
$$

An important length scale is also the de Broglie wavelength $\Lambda_{i} \equiv\left(2 \pi \hbar^{2} / m_{i} k_{B} T\right)^{1 / 2}$, where $m_{i}$ is the mass of ion of species $i$.

In the high density and degenerate plasma limit, i.e. $r_{s} \rightarrow 0$ and $\Theta \rightarrow 0$ respectively, the electrons form a rigid neutralizing background. However, for most of the conditions the electron background is both polarizable and compressible. In charged 
fluids the electrons interact not only with their immediate neighbors but with all the other electrons in the system, therefore their motion cannot be decoupled from one another. As a consequence plasmas exhibit a strong collective behavior in the long wavelength limit, $k \rightarrow 0$, where the Fourier transform of the Coulomb potential, $e^{2} / \varepsilon_{0} k$, diverges. A manifestation of this behavior is the rearrangement of the electrons around the ions so that the plasma remains locally neutralized. This leads to an effective ion potential which decays much faster than $1 / r$ at large distances. In this work we use the Yukawa potential to describe the ionic effective potential.

To get an insight into the diffusion of dense plasmas we will study a binary ionic mixture immersed in a polarizable background of electrons that adiabatically cloud around the ions. Conceptually, although cumbersome, we can extend the treatment of a binary mixture to a multicomponent mixture. We touch on this topic for the interested reader in the appendix 6 . We denote the mass fraction of component $\alpha$ by $c_{\alpha} \equiv \rho_{\alpha} / \rho$, where $\rho$ and $\rho_{\alpha}$ are the mass density of the total mixture and this component, respectively. 


\section{General overview of diffusivity in mixtures}

Species diffusivity as a response to the concentration gradient is the main contributor to how fluid evolves with time. Other contribution come from pressure and thermal gradients in a way that respects conservation of mass, energy momentum and even number of particles if chemical reactions are ignored. The conservation laws and dissipative fluxes are described by the Navier-Stokes equations for multicomponent fluids. The complete set of these equations is complicated and given in standard texts [13]. Here we consider the binary mixture case and write only equations that are relevant to the diffusion. The conservation equations for mass and momentum and the equation for the species flux are:

$$
\begin{gathered}
\frac{\mathfrak{D} \rho}{\mathfrak{D} t}=-\rho \nabla \cdot \mathbf{v}, \\
\rho \frac{\mathfrak{D} c}{\mathfrak{D} t}=-\nabla \cdot \mathbf{J}+r, \\
\rho \frac{\mathfrak{D} \mathbf{v}}{\mathfrak{D} t}=-\nabla p-\nabla \cdot \tau+\rho \mathbf{g}, \\
\mathbf{J}=-\rho D_{12}\left[\nabla c+\left(k_{T} / T\right) \nabla T+\left(k_{p} / p\right) \nabla p\right],
\end{gathered}
$$

where $\mathfrak{D} f / \mathfrak{D} t$ is the comoving derivative (e.g., $\mathfrak{D} \rho / \mathfrak{D} t=\partial_{t} \rho+\mathbf{v} \cdot \nabla \rho$ ). In these sets of equations $\mathbf{v}(\mathbf{r})$ is the velocity field, $\rho(\mathbf{r})$ is density and $c(\mathbf{r})$ is the mass fraction. The equations are written for a binary mixture so the indices in the previous definition have been dropped. Also $\mathbf{g}$ is the gravitational acceleration, $D_{12}$ is the interdiffusivity, $k_{T} D_{12}$ is the thermal diffusion coefficient and $k_{P} D_{12}$ is the barodiffusion coefficient.

Here $\tau_{i j}$ is the energy-momentum tensor related to the stress tensor $\sigma_{i j}$ by $\tau_{i j}=$ $\rho v_{i} v_{j}-\sigma_{i j}$. The rate of production of mass of one species due to chemical reaction, which will be ignored in this study, is denoted by $r$. The other fields are the internal energy per unit mass $E$, pressure $\mathbf{p}=-\frac{1}{3} \sigma_{i i}$, and temperature $T$.

In this work we are interested in Fick's law, which is implied by Eqs. (9) and 11. We consider cases with no temperature, density and pressure gradient. Below we describe the self-diffusion and its relation to the Fickian diffusion.

\subsection{Self-Diffusivity}

The self-diffusion coefficient $D_{\alpha}(\alpha=1,2)$ is related to the random-walk motion of a tagged particle of species $\alpha$ in a mixture on hydrodynamic scales. It can be calculated from the velocity autocorrelation function [24, 25, 26, 27, 1, 28]: 


$$
C_{\alpha}(t)=\frac{1}{3 N_{\alpha}} \sum_{j=1}^{N_{\alpha}}\left\langle\mathbf{v}_{j}^{(\alpha)}(t) \cdot \mathbf{v}_{j}^{(\alpha)}(0)\right\rangle
$$

via a Green-Kubo integral,

$$
D_{\alpha}=\int_{0}^{\infty} C_{\alpha}(t) \mathrm{d} t
$$

In Eq. $12, \mathbf{v}_{j}^{(\alpha)}(t)$ is the velocity of particle $j$ of species $\alpha$ at time $t$. The autocorrelation function $C_{\alpha}$ depends on the species as well as temperature, pressure, density and composition. The autocorrelation starts at $C_{\alpha}(0)=k_{B} T / m_{\alpha}$ and decreases toward 0 with time. For moderate to strong coupling mixtures the autocorrelation function exhibits an oscillatory decay with time due to the appearance of negative correlation effects. This is a manifestation of the cage effect [29]; where the tagged particle finds itself momentary trapped by its immediate neighbors. At low enough coupling the autocorrelation function decays monotonically; an indication of the absence of the aforementioned many-body effects.

It is straightforward to verify the equivalence of Eq. 13 with the equation relating the mean-squared displacement of a tagged diffusing particle time, in the long time limit. This is given by the well known relation due to Einstein for Brownian motion [30]:

$$
D_{\alpha}=\lim _{t \rightarrow \infty} \frac{1}{6 t}\left\langle|\mathbf{r}(t)-\mathbf{r}(0)|^{2}\right\rangle .
$$

We can rewrite the Einstein relation (14) in terms of velocity autocorrelation as follows [24, 25]:

$$
\left\langle|\mathbf{r}(t)-\mathbf{r}(0)|^{2}\right\rangle=\left\langle\int_{0}^{t} d t^{\prime} \mathbf{v}\left(t^{\prime}\right) \cdot \int_{0}^{t} d t^{\prime \prime} \mathbf{v}\left(t^{\prime \prime}\right)\right\rangle=2 \int_{0}^{t} d t^{\prime} \int_{0}^{t^{\prime}} d t^{\prime \prime}\left\langle\mathbf{v}\left(t^{\prime \prime}\right) \cdot \mathbf{v}\left(t^{\prime}\right)\right\rangle
$$

Equation (15) can be combined with the definition (12) to give:

$$
\left\langle|\mathbf{r}(t)-\mathbf{r}(0)|^{2}\right\rangle=6 \int_{0}^{t} d t^{\prime} \int_{0}^{t^{\prime}} d t^{\prime \prime} C\left(t^{\prime}-t^{\prime \prime}\right) .
$$

By changing variables from $t^{\prime}$ and $t^{\prime \prime}$ to $t^{\prime}$ and $\tau \equiv t^{\prime}-t^{\prime \prime}$ and after integration by parts, we find that:

$$
\left\langle|\mathbf{r}(t)-\mathbf{r}(0)|^{2}\right\rangle=6 t \int_{0}^{t}\left(1-\frac{\tau}{t}\right) C(\tau) d \tau .
$$

Substitution of Eq. (17) into (14) verifies (13). This equivalence is a direct consequence of Fick's law of diffusion and constitutes a simple example of the general fluctuation - dissipation relation, also known as Green-Kubo relations [31, 32, 33, 34]. It also relates the stochastic process of a random walk in which the mean square displacement of the walker becomes a linear function of time after a sufficiently larger number of collisions and displacements have occurred. 


\subsection{Interdiffusion}

Interdiffusion is related to the collective transport of mass driven by concentration gradients [24]. The rate at which concentration fluctuations dissipate is governed by the interdiffusion coefficient $D_{12}$ which linearly relates mass fluxes to gradients in the chemical potential. The latter, in isobaric-isothermal conditions, is linearly proportional to the gradient of mass concentration. This can be easily seen from equation (11) by keeping only the first term inside the bracket in the RHS. If we define the center of mass velocity field by:

$$
\mathbf{v}(\mathbf{r}, t)=\sum_{\alpha=1}^{2} c_{\alpha} \mathbf{v}_{\alpha}(\mathbf{r}, t)
$$

where $\mathbf{v}_{\alpha}$ is the velocity field of species $\alpha$, then the mass flux of this species is :

$$
\mathbf{J}_{\alpha}(\mathbf{r}, t) \equiv \rho_{\alpha}(\mathbf{r}, t)\left[\mathbf{v}_{\alpha}(\mathbf{r}, t)-\mathbf{v}(\mathbf{r}, t)\right]
$$

and we define the interdiffusion coefficient by the Fick's law:

$$
\mathbf{J}_{\alpha}(\mathbf{r}, t)=-\rho(\mathbf{r}, t) D_{12} \nabla c_{\alpha}(\mathbf{r}, t)
$$

Using molecular dynamics we can study and estimate the interdiffusion by creating a system, like the one in Fig (1), with an initial sharp interface that creates a very steep composition gradient. From the Fick's law a current will be created that opposes this gradient and with time broadens the interface, and therefore intermixing the different components. This process is very clear in Fig. 1 where we have simulated the mixing process between two plasma of argon (in gold color on right) and deuterium (in silver color on left). In principle, one can estimate the interdiffusivity $D_{12}$ by analyzing the evolution of the composition profile $c_{\alpha}(\mathbf{r}, t)$ which should satisfy the Fick's equation at longtime limit. At the very initial stage when strong gradients are present non-Fickian effects, like diffusion from ballistic motion, occur.

The interdiffusion coefficient like the self-diffusion can be expressed in terms of fluctuations in the system in equilibrium, without any external forces to drive mass or energy flux $([24,25,35,1])$, by use of Green-Kubo techniques [31]. In the following we briefly derive the appropriate Green-Kubo formalism by connecting with Fick's law in the low-frequency and long-wavelength fluctuations that correspond to the regime governed by linear hydrodynamics. A more detailed derivation can be found in classical books of Statistical Mechanics [24, 25]. We also provide a general derivation of this technique for a more generic case of multicomponent mixtures in appendix 6. We consider only mass concentration fluctuations and ignore fluctuations in temperature and pressure. Using the previous notation and the overall mass conservation law, one component should satisfy:

$$
\rho(\mathbf{r}, t)\left(\frac{\partial c_{1}(\mathbf{r}, t)}{\partial t}+\mathbf{v}(\mathbf{r}, t) \cdot \nabla c_{1}(\mathbf{r}, t)\right)=-\nabla \cdot \mathbf{J}_{1}(\mathbf{r}, t) .
$$



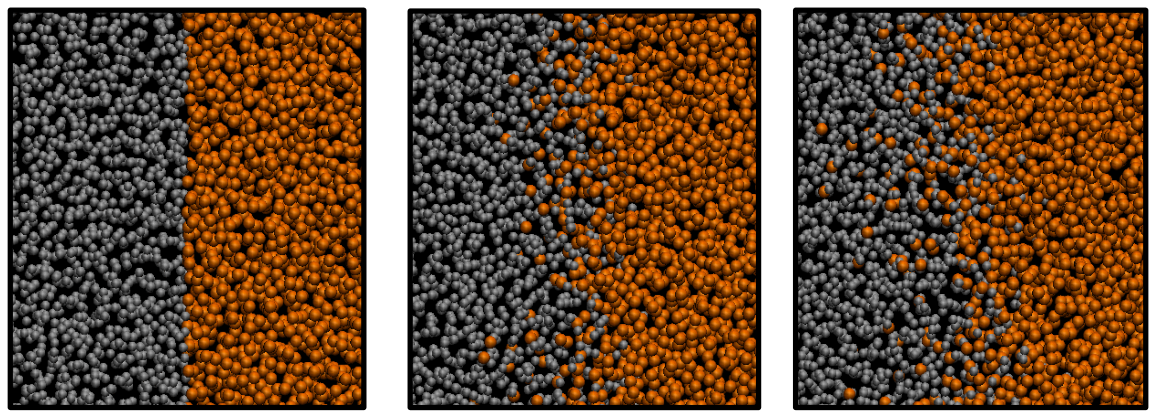

Fig. 1: A non-equilibrium molecular dynamics simulation for binary mixtures of deuterium and argon. In this plot chronologically ordered snapshots of interface broadening between two plasma $\mathrm{Ar}$ and $\mathrm{D}$ as they mix are shown.

Linearizing the above equation with respect to virtual mass fluctuation of species 1 , $\delta c_{1}(\mathbf{r}, t)$ we arrive at the Fick's diffusion equation for this fluctuation:

$$
\frac{\partial}{\partial t} \delta c_{1}(\mathbf{r}, t)=D_{12} \nabla^{2} \delta c_{1}(\mathbf{r}, t) .
$$

It is very instructive to relate the fluctuations in local mass concentration to the fluctuations in local number densities by the following ( cf. Ref. [24], Ch.8):

$$
\delta c(\mathbf{r}, t)=\frac{m_{1} m_{2}}{\rho^{2}}\left[n_{2} \delta n_{1}(\mathbf{r}, t)-n_{1} \delta n_{2}(\mathbf{r}, t)\right]
$$

The real space solution of Eq. 22] in terms of Fourier components is:

$$
\delta c_{1}(\mathbf{k}, t)=\delta c_{1}(\mathbf{k}, 0) \exp \left(-D_{12} k^{2} t\right) .
$$

Ensemble averaging of the correlation of the above fluctuations followed by a Laplace transform in time, results in the following relation :

$$
\begin{aligned}
\left\langle\delta c_{1}(-k, \omega) \delta c_{1}(k, t=0)\right\rangle & \equiv \int_{0}^{\infty} e^{i \omega t}\left\langle\delta c_{1}(-k, t) \delta c_{1}(k, t=0)\right\rangle d t \\
& =\left\langle\left|\delta c_{1}(k, t=0)\right|^{2}\right\rangle \frac{i \omega+D_{12} k^{2}}{\omega^{2}+D_{12}^{2} k^{4}} .
\end{aligned}
$$

By taking the appropriate hydrodynamic limit (i.e., long wavelength and low frequency limit) and after use of mass conservation and integration by parts [36] we get the following Green-Kubo form for interdiffusion coefficient:

$$
D_{12}=\frac{1}{3 \lim _{k \rightarrow 0} S_{c c}(k)} \int_{0}^{\infty} d t\left\langle\mathbf{j}^{c}(t) \cdot \mathbf{j}^{c}(0)\right\rangle .
$$


In the above $S_{c c}$ is the concentration structure factor defined in terms of partial structure factors $S_{i j}(k)=\frac{1}{N} \sum_{\alpha=1}^{N_{i}} \sum_{\beta=1}^{N_{j}}\left\langle\exp i \mathbf{k} \cdot\left(\mathbf{r}_{\alpha}-\mathbf{r}_{\beta}\right\rangle\right.$, with $i, j$ the species indices, by the following relation due to Bhatia \& Thornton [37]:

$$
S_{c c}(k)=X_{2}^{2} S_{11}(k)+X_{1}^{2} S_{22}(k)-2 X_{1} X_{2} S_{12}(k)
$$

The expression 26 for interdiffusivity can simply be written as a product of a thermodynamic factor $\Phi$ with a Green-Kubo integrand $D_{12}^{0}$ :

$$
D_{12}=\Phi D_{12}^{0}
$$

Below we briefly discuss about each of these factors.

\subsubsection{Thermodynamic Factor}

The thermodynamic factor in Eq. 28 is defined by $\Phi \equiv X_{1} X_{2} / S_{c c}(0)$. In the long wavelength limit all the structure factors can be derived by either thermodynamic fluctuation theory or by calculation in a grand canonical ensemble [38]. The concentration structure factor in isobaric-isothermal conditions is given by:

$$
S_{c c}(0)=1 /\left(\frac{\partial^{2}(\beta G / N)}{\partial X_{1}^{2}}\right)_{P, T} .
$$

Therefore we can express the thermodynamic factor as:

$$
\Phi=X_{1} X_{2}\left[\frac{\partial^{2}(\beta G / N)}{\partial X_{1}^{2}}\right]_{P, T},
$$

where $G$ is the Gibbs free energy, $\beta \equiv k_{B} T$ and $N$ is the total number of particles in the system.

In most of the cases for nearly ideal neutral mixtures the thermodynamic factor is very close to unity; however, this may not be true when considering charged plasmas. For weakly coupled plasmas due to the ambipolar electric field of the electrons this factor goes to $\left\langle Z^{2}\right\rangle /\langle Z\rangle^{2}$, which can be very large at low concentration of an asymmetric mixture [20, 35, 39, 40]. As an example of this effect in Fig. 2] we show the variation of this factor as a function of the mole fraction of the heaviest particle in a binary mixture for four cases of charge ratio of the components, namely, $Z_{2} / Z_{1}=4,8,13,18$. Note that the asymmetry of the mixture is characterized by this ratio. As it is clear in low mole fraction the higher the asymmetry the further from unity is the thermodynamic factor.

Interestingly, significant deviations of the thermodynamic factor $\Phi$ from unity has been recently observed numerically for non-plasma mixture of Al-Ni melts [27]. 


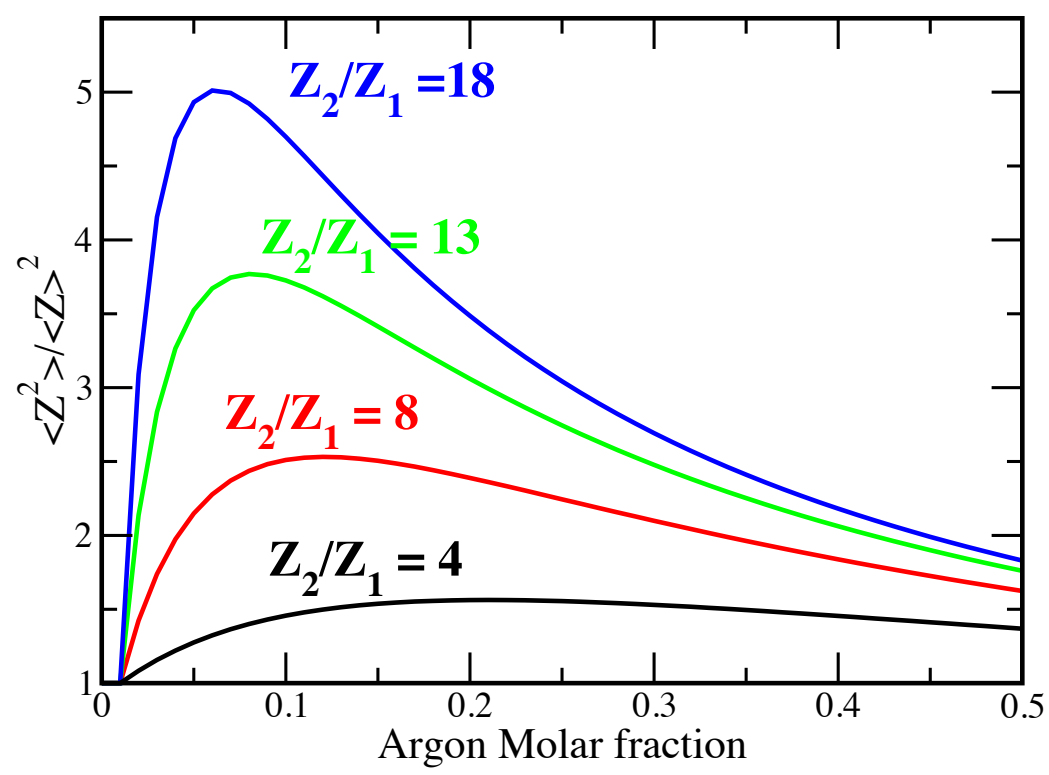

Fig. 2: The thermodynamic factor $\left\langle Z^{2}\right\rangle /\langle Z\rangle^{2}$ as a function of mole fraction for four different ratios of component charge $Z_{2} / Z_{1}=4,8,13,18$.

\subsubsection{Green-Kubo: Maxwell-Stefan Diffusivity}

The Green-Kubo integrand Eq. 287 is given by:

$$
D_{12}^{0}=\frac{1}{3 N X_{1} X_{2}} \int_{0}^{\infty} d t\left\langle\mathbf{j}^{c}(t) \cdot \mathbf{j}^{c}(0)\right\rangle
$$

where the term inside the integral is a correlation of interdiffusivity currents:

$$
\mathbf{j}^{c}(t)=X_{2} \sum_{i=1}^{N_{1}} \mathbf{v}_{i}-X_{1} \sum_{j=1}^{N_{2}} \mathbf{v}_{j}=N X_{1} X_{2}\left(\bar{v}_{1}-\bar{v}_{2}\right)
$$

The above Green-Kubo integrand $D_{12}^{0}$ is also known in literature as Maxwell-Stefan diffusivity [41].

\subsection{Relating Self-diffusion with Interdiffusion}

The velocity correlation functions in the interdiffusion are collective: only one degree of freedom, the difference between the center of mass velocities for the two species, contributes to the autocorrelation function whereas the self-diffusivity is 
related to the velocity autocorrelation function of the individual particles. Instead of one degree of freedom, the average is over $N_{i}$ degrees of freedom. The statistics are much better for calculating self-diffusivity. This difference explains why in molecular dynamics simulations it is difficult to achieve the same numerical precision when estimating Maxwell-Stefan diffusivity as compared to self-diffusion coefficient [1, 42, 43, 44]. Therefore, it is extremely helpful to identify practical relations between Maxwell-Stefan and self-diffusion through different simplifying assumptions.

If we group separately the velocity autocorrelation and cross-correlation functions that appear in Maxwell-Stefan diffusion coefficient (31) and use the $\mathscr{F}$ notation of McCall and Douglass [45, 28, 46], we have the following:

$$
D_{12}^{0}=\left[X_{2} D_{1}+X_{1} D_{2}+X_{1} X_{2}\left(\frac{\mathscr{F}_{11}}{X_{1}^{2}}+\frac{\mathscr{F}_{22}}{X_{2}^{2}}-2 \frac{\mathscr{F}_{12}}{X_{1} X_{2}}\right)\right],
$$

where the $\mathscr{F}$-factors are given by:

$$
\mathscr{F}_{\alpha \beta}=\frac{1}{3 N} \sum_{i=1}^{N_{\alpha}} \sum_{j \neq i}^{N_{\beta}} \int_{0}^{\infty}\left\langle\mathbf{v}_{i}(t) \cdot \mathbf{v}_{j}\right\rangle d t .
$$

If we assume that velocity cross-correlation functions are negligible, i.e.,

$$
\frac{\mathscr{F}_{11}}{X_{1}^{2}}+\frac{\mathscr{F}_{22}}{X_{2}^{2}}-2 \frac{\mathscr{F}_{12}}{X_{1} X_{2}}=0
$$

a simple linear rule that connects Maxwell-Stefan with self-diffusion coefficient in a binary system follows:

$$
D_{12}^{0}=X_{2} D_{1}+X_{1} D_{2} .
$$

The above relation is known in the Condensed Matter Community as Darken's equation [47, 48, 46, 49]. Similar relation has tacitly been assumed also in the plasma community [42, 35, 22, 23].

A similarity exists between Eq. (36) and the so-called Nernst-Einstein relation, which is an empirical relation that links the electrical conductivity of an ionic system to the self-diffusion coefficients of the cations and anions in the system [50]. In this case the electrical conductivity is related to the same velocity correlation functions as the one that expresses the interdiffusion coefficient. 


\section{Diffusion models in plasmas from Kinetic Theories}

Plasmas, whether in astrophysics or in the Inertial Confinement Fusion experiments, span a huge range of the Coulomb coupling parameter $\Gamma$ (6). In the weakly coupled regime Chapman-Spitzer theories $([51,14])$ have been very successful in describing diffusion coefficient.

In the limit of dilute-gas approximation transport coefficients, including diffusivity, are estimated by considering the Boltzmann equation of kinetic theory. Here the collisions are important in determining the distribution function of the particles. In weakly coupled plasmas ion-ion collisions consist of a series of many small angle binary scattering events. Within these assumptions the Chapman-Enskog theory [14] assumes that the total distribution function of a given species can be expanded as a convergent series of functions with the lowest order corresponding to the equilibrium distribution. Transport coefficients are then calculated from velocity moments of the velocity distribution function. The latter is further expanded in terms of Sonine polynomials (Associated Laguerre Polynomials). Having only the first term in this expansion will correspond to a Maxwell velocity distribution. In this first polynomial approximation following Chapman and Cowling [14], the interdiffusion coefficient is given by:

$$
\left[D_{12}\right]_{1}=\frac{3 k_{B} T}{16 n m_{r e d} \Omega_{12}^{(11)}},
$$

where $T$ and $n$ are temperature and ion density, respectively, and

$$
m_{\text {red }} \equiv \frac{m_{1} m_{2}}{m_{1}+m_{2}}
$$

is the reduced mass of particles each with mass $m_{1}$ and $m_{2}$. Here $\Omega_{12}^{(11)}$ is the first collision integral -defined in appendix 6- which depends on the exact nature of the interaction of colliding particles. For a pure Coulomb interaction between two particles with charge $Z_{1}$ and $Z_{2}$ this integral can be derived analytically [14] as:

$$
\Omega_{12}^{(11)}=\frac{1}{4 \pi \varepsilon_{0}} \sqrt{\frac{\pi}{32}} \frac{Z_{1}^{2} Z_{2}^{2} e^{4}}{\sqrt{m_{\text {red }}}\left(k_{B} T\right)^{3 / 2}} \log \left(1+4 \pi \varepsilon_{0} \frac{16\left(k_{B} T\right)^{2} \lambda_{D}^{2}}{Z_{1}^{2} Z_{2}^{2} e^{4}}\right) .
$$

The Debye length $\lambda_{D}$ is given by:

$$
\lambda_{D}=\sqrt{\frac{\varepsilon_{0} k_{B} T}{e^{2} \sum X_{i} Z_{i}^{2}}}=\sqrt{\frac{\varepsilon_{0} k_{B} T}{e^{2}\left\langle Z_{i}^{2}\right\rangle}},
$$

where at the last term we have used the molar weighted average convention for the $Z^{2}$, with $X_{i}$ being the mole fraction of species $i$.

Accounting for a second-order polynomial approximation resulting from deviations from Maxwell distributions provides: 


$$
\left[D_{12}\right]_{2}=\frac{\left[D_{12}\right]_{1}}{1-\Delta}
$$

where $\Delta$ is a function of the collision integrals $\Omega_{12}^{(11)}, \Omega_{12}^{(12)}, \Omega_{12}^{(13)}, \Omega_{12}^{(22)}$ and the mole fraction of the mixture. Details of its expression can be found at [15]. Inclusion of terms beyond second polynomial introduce very small correction to the value of diffusivity.

The use of an effective screened Coulomb interaction gives, in principle, a better description of the diffusion in plasmas as it accounts for the collective effects that surround a given charge. The collision integrals for this potential have been numerically calculated and tabulated by Paquette et al. [15]. Using these tabulated collision integrals and a second polynomial expansion we plot in Fig. 3 the diffusivity values for a binary deuterium-argon mixture as a function of the mole fraction, for three different $T=100,500$ and $1000 \mathrm{eV}$ and, ion density $n=10^{25} / \mathrm{cm}^{3}$. In all these calculations the ionization of deuterium and argon was kept at values 1 and 13 , respectively.

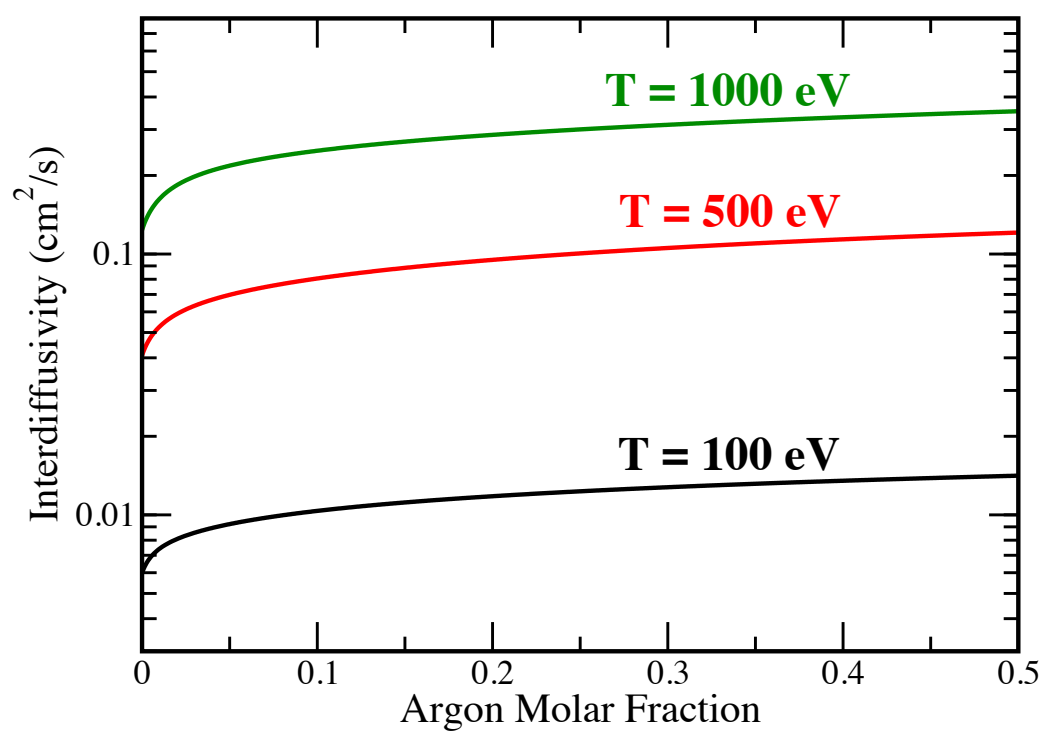

Fig. 3: Interdiffusivity results for a deuterium-argon mixture at three different temperatures with ion density $n=10^{25} / \mathrm{cm}^{3}$. The values were calculated by using the 2 nd polynomial approximation and the tabulated collision integrals for screened Coulomb interaction [15]. In all these calculations the ionization of deuterium and argon was kept at values 1 and 13, respectively. 


\section{Methods}

An extremely powerful, albeit simple, tool to calculate diffusion coefficients is molecular dynamics (MD) simulation. MD does not make weak coupling or binary collision approximations, so it allows the study of diffusion with strong coupling. MD simulations follow the motion of atoms (ions) by integrating their equations of motion while accounting for the pairwise particle-particle interactions [52, 53]. The ions move according to the Newton's Second Law:

$$
m \ddot{\mathbf{r}}_{i}=\sum_{i \neq j}^{N} \mathbf{F}_{i, j}(t)
$$

This set of $3 N$ coupled ordinary differential equations are integrated explicitly in time using the leap-frog scheme or velocity Verlet [52] algorithm, with a time step that is related to the characteristic time scale of the system. Here $N$ is the total number of particles in the system. In Eq. (42), $\mathbf{F}_{i, j}$ is the force originating from the interaction of "test" particle $i$ with particle $j$. We study an unconfined system of particles which entails use of periodic boundary conditions (PBC).

We use this general tool to study our plasma system as a mixture of deuterium (D) and $\operatorname{argon}(\mathrm{Ar})$. At a temperature $T=100 \mathrm{eV}$ we assume that D is fully ionized while Ar partially ionized. The ionization of Ar has been input as a free parameter in order to study its effect. Since the system that we are considering has ions, the interaction force $\mathbf{F}_{i, j}$ among these ions has a Coulombic nature. The presence of free electrons, that act as a neutralizing background, results in an effective ion-ion interaction potential. Within linear response theories [54, 55] the interaction between ions is the Yukawa potential:

$$
\phi\left(\mathbf{r}_{i j}\right)=\frac{Z_{i} Z_{j} e^{2}}{4 \pi \varepsilon_{0} r_{i j}} \exp \left(-r_{i j} k_{D}\right),
$$

where $1 / k_{D}$ is the screening length due to the electrons.

In the following we give a succinct description on how this screening arises and how it is related to the thermodynamic conditions. Following the Born-Oppenheimer approximation, the adiabatic separation of motions of heavy and light charged particles leads to an effective ion-ion interactions, or heavy-heavy particle, with the electrons, the light particles, forming a cloud around, and instantaneously following, the ions. To derive this effective potential between the ions we calculate the electron density distribution in space when the ions are embedded in their midst. The potential $\phi(\mathbf{r})$ that gives rise to the effective interaction can be derived from the Poisson equation:

$$
\nabla^{2} \phi(\mathbf{r})=\frac{e}{\varepsilon_{0}}\left[\sum_{i} Z_{i} \delta\left(\mathbf{r}-\mathbf{r}_{\mathbf{i}}\right)-n_{e}(\mathbf{r})\right]
$$

with the electron density $n_{e}$ satisfying the following normalization condition: 


$$
2 \int f(\mathbf{p}) \frac{4 \pi p^{2} d p}{(2 \pi \hbar)}=n_{e}(\mathbf{r})
$$

The factor 2 in front of the integral accounts for the two spin states of the electron: up and down. The electron follow the Fermi-Dirac statistics, therefore $f(\mathbf{p})$ is the electron distribution function in momentum space given by:

$$
f(\mathbf{p})=\frac{1}{\exp \left(\frac{p^{2} / 2 m_{e}-\mu_{e}}{k_{B} T}\right)+1},
$$

where $\mu_{e}$ is the chemical potential of the electron.

The presence of this field, or equivalently the ions, varies the electron density as $\Delta n_{e}(\mathbf{r})=n_{e}\left(\mu_{e}+e \phi(\mathbf{r})\right)-n_{e}\left(\mu_{e}\right)$. Within linear response theory [54] this change is: $\Delta n_{e}(\mathbf{r})=-e^{2} \frac{\delta n_{e}}{\delta \mu_{e}} \phi(\mathbf{r})$. Plugging this into Eq. 44 gives $\phi(\mathbf{r})$ as the Yukawa potential Eq. 43 with a screening coefficient $k_{D}=-e^{2} \frac{\delta n_{e}}{\varepsilon_{0} \delta \mu_{e}}$. This coefficient can be exactly derived in two extreme cases:

- In the limit of non-degenerate electrons $\Theta \rightarrow \infty$, i.e., very high $\mathrm{T}$ and low density, the electrons satisfy the Maxwell-Boltzmann statistics which results in the Debye-Hückel [56] screening coefficient:

$$
k_{D}=\sqrt{\frac{n_{e} e^{2}}{\varepsilon_{0} k_{B} T}} .
$$

This corresponds to the classical limit.

- In the fully degenerate limit $\Theta \rightarrow 0$, i.e., very low $\mathrm{T}$ and high density, which corresponds to an electron gas in the ground state [57], the screening coefficient is given by:

$$
k_{D}=\sqrt{\frac{3}{2} \frac{n_{e} e^{2}}{\varepsilon_{0} E_{F}}} .
$$

At finite temperatures and densities we can use interpolation forms, like the one from Itchimaru [58, 59]

$$
\frac{\mu_{e}}{k_{B} T}=\frac{3}{2} \ln \Theta+\ln \frac{4}{3 \sqrt{\pi}}+\frac{A \Theta^{-b-1}+B \Theta^{-(b+1) / 2}}{1+A \Theta^{-b}},
$$

with $A, B$ and $b$ coefficients given at [59]. A more practical interpolation for numerical studies of transport coefficients, which we adopt for the rest of this work, is the one introduced by Murillo [60]:

$$
k_{D}=\sqrt{\frac{\langle Z\rangle n e^{2}}{\varepsilon_{0} \sqrt{\left(k_{B} T^{2}\right)+\left(\frac{2}{3} E_{F}\right)^{2}}}}
$$


Note above that we have used Eq. (5) to relate electron density $n_{e}$ with ion density $n$ and the average charge $\langle Z\rangle$ of the mixture.

The screening length sets the length scale of the system. The system that interacts through Yukawa potential can be described by the dimensionless screening coefficient $\kappa=a_{W S} k_{D}[16]$ and the effective coupling $\Gamma_{\text {eff }}$. When the screening coefficient $\kappa$ is large the Yukawa potential is a short range potential. In this case the MD methods make use of the truncation of the interaction potential therefore limiting the need for the summation of pairwise interactions around a test particle to a region of finite size. In the case of long range interaction, like weak screening Yukawa or Coulomb interaction, such truncation of the potential is not allowed and special techniques, like Ewald [61] summation, have to be used in MD simulations. A more efficient method is to use the so called particle-particle particle-mesh (PPPM) scheme [62, 63], where the interparticle force is partitioned into: (i) a shortrange particle-particle force which is to be applied to closely separated ions only, and (ii) a force component that can be calculated on the mesh. In the mesh part of the calculation charged clouds are used instead of point-like particles and their interactions is calculated on a computational mesh taking into account periodic images. This method makes it possible to treat long-range and low- $\kappa$ Yukawa potentials. For screening values $\kappa \geq 1$, the particle-particle part alone provides sufficient accuracy while being numerically more efficient. In these cases the mesh part need not to be used and the interaction forces are summed for particles situated within a ( $\kappa$-dependent) cutoff radius around the particle.

The simulations presented here are initiated from a spatially random particle configuration, and particle velocities sampled from a Maxwellian distribution of temperature $T=100 \mathrm{eV}$. This random configuration of the particles of each component ensures a spatially uniform mixture as well, suitable for the equilibrium MD. At the initial stage of the run the system is equilibrated to the desired temperature by either (i) rescaling the particle momenta or (ii) by applying Nosé-Hoover [64, 65, 52] thermostats to keep the system initially at a constant number of particles $\mathrm{N}$, volume $\mathrm{V}$ and temperature T. On average the system is left to equilibrate for 50000 timesteps in the NVT ensemble. This is followed by the production run in a microcanonical ensemble with constant $\mathrm{N}$, volume $\mathrm{V}$ and total energy $\mathrm{E}$.

The time scale of the system is set by the ionic plasma frequency :

$$
\omega_{p}=\sqrt{\frac{\langle Z\rangle^{2} n}{\varepsilon_{0}\langle m\rangle}} .
$$

The timestep in most of the runs was taken $\Delta t \simeq 1 /\left(100 \omega_{E}\right)$, where $\omega_{E}$ is the Einstein frequency, that physically describes the oscillatory motion of a caged particle in the well potential created by its neighbors. In the limit of no screening $\kappa \rightarrow 0$ we have $\omega_{E}=\omega_{p} / 3$ [16], and for a finite screening we use a fitted form $\omega_{E}(\kappa)=\omega_{p} \exp \left(-0.2 \kappa^{1.62}\right)$ [60] to the Ohta and Hamaguchi MD results [16].

To calculate the self-diffusion coefficients of a binary mixed plasma of $\mathrm{D}$ and $\mathrm{Ar}$ we have performed MD simulations in the following range of conditions: temperature $T=100 \mathrm{eV}$, particle number density $n=10^{23} / \mathrm{cm}^{3}$ and $10^{25} / \mathrm{cm}^{3}$, fully ionized 
deuterium and ionization of $\operatorname{Ar} Z_{A r}=4,8,13$ and 18. The last of $Z_{A r}$ will correspond to a fully ionized Ar. We take the ionization of $\mathrm{Ar}$ as a free parameter; for reference, an average atom model [66] gives the Ar ionization as $Z_{A r} \simeq 10.5$ at $T=100 \mathrm{eV}$, $n=10^{25} / \mathrm{cm}^{3}$ and $X$ in the range $0.01-0.5$. Specifically, for each of the above conditions we consider mixtures whose Ar mole fraction is: $X=0.01,0.05,0.1,0.2$ and 0.5 . The calculations were performed with enough particles $(30000 \geq N \geq 120000)$ over long enough time scales to ensure convergence with insignificant statistical uncertainty.

The autocorrelation function was calculated on the fly as the simulation progressed:

$$
C_{i}(t)=\frac{1}{3 N_{i}} \sum_{\alpha \in i} \mathbf{v}_{i}(t) \cdot \mathbf{v}_{i}(0),
$$

where $i$ indicates the type of the atom and $N_{i}$ its numbers. For each atom the initial velocity $\mathbf{v}(0)$ is saved. The simulation was run on parallel supercomputers and so the initial velocity together with other atom attributes has to be communicated when an atom has to move from a spatial domain related to a processor into another. The correlation velocity is saved as a table for a sufficiently large time span. Following Rudd et al. [1], the self-diffusivity was then calculated as an integral over the velocity autocorrelation function by postprocessing:

$$
\begin{aligned}
D_{i} & =\int_{0}^{\infty} d t C_{i}(t) \\
& \approx\left[\sum_{k} a_{k} \Delta t C_{i}\left(t_{k}\right)\right]+\frac{t_{\max }}{\alpha-1} C_{i}\left(t_{\max }\right),
\end{aligned}
$$

where the coefficients $a_{k}$ give the Simpson's rule approximation to the integral. The integral was terminated to account for a power law $t^{-\alpha}$ decay in the correlation function [1]. In particular the long-time tail contribution was included through this term. For the conditions that we consider in our simulations the tail contribution was small (less than 5\%). 


\section{Results}

We have used MD to calculate the velocity autocorrelation functions and selfdiffusivities across a broad range of conditions for a binary ion mixture of deuterium and argon in the presence of a polarizable electron background. The ion-ion interaction is the Yukawa potential (screened Coulomb) Eq. 43, that incorporates the effect of the electrons in the screening coefficient. The latter is calculated using Eq. [50] [60]. In these simulations we have used ionization as an input parameter in order to see its effects along with temperature, density and composition on diffusivity.

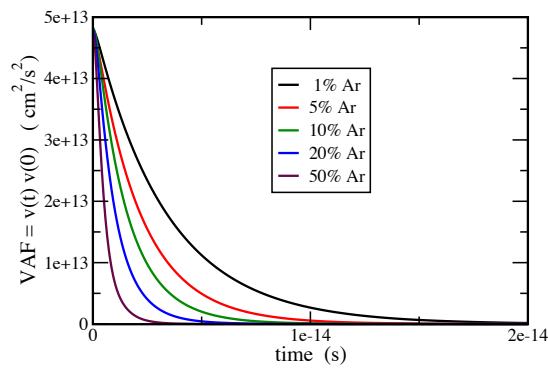

(a) Velocity Autocorrelation Function of deuterium

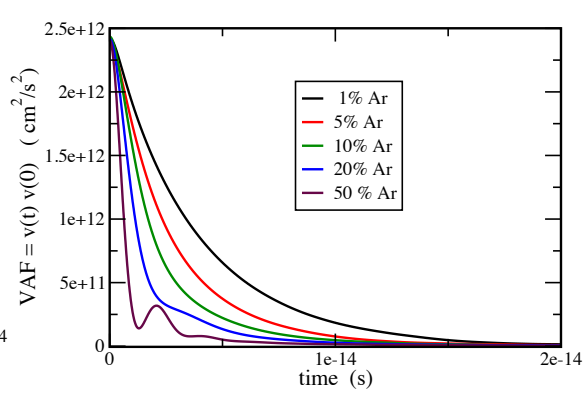

(b) Velocity Autocorrelation Function of argon

Fig. 4: Velocity autocorrelation function using molecular dynamics as a function of time for five different mole fraction of argon, namely $X=0.01,0.05,0.1,0.2,0.5$. In these different mixtures we consider a constant ionization for deuterium and argon at $Z_{D}=1$ and $Z_{A r}=13$, respectively. These mixtures were kept at temperature $T=100 \mathrm{eV}$ and number density $n=10^{25} / \mathrm{cm}^{3}$.

Figure 4 shows calculation from MD simulations of the velocity autocorrelation function of deuterium $C_{D}$ on the left, and of argon $C_{A r}$ on the right as a function of time. Here different lines correspond to results from mixtures with different $\mathrm{Ar}$ mole fraction, namely $X=0.01,0.05,0.1,0.2$ and 0.5 . In these plots the temperature was kept at $T=100 \mathrm{eV}$ and the ionization of deuterium and argon at $Z_{D}=1$ and $Z_{A r}=13$, respectively. As we changed the composition we kept the ion density constant at $n=10^{25} / \mathrm{cm}^{3}$ which entails a change of mass density. A change from a $1 \%$ to $50 \%$ argon mole fraction, is paralleled with a change in mass density from $\rho=39.517$ to $348.547 \mathrm{~g} / \mathrm{cm}^{3}$.

For mixtures with higher argon mole fraction, the screening coefficient $\kappa$ as well as the couplings; $\Gamma_{e f f}, \Gamma_{A r}$ and $\Gamma_{D}$, defined in Eqs (7) and (6), are increased. For low to moderate coupling values the many-body correlation effects are not very pronounced. As a consequence the autocorrelation function decreases to 0 monotonically with time. On the other hand the autocorrelation function decays faster to 0 when the coupling value is higher. 
As the coupling is increased-in this case by increasing the amount of $\mathrm{Ar}$-we notice the formation of structures in the autocorrelation function. This is due to the so-called "cage-effect" [67, 29], where the motion of the tagged particle is correlated in a temporary cage of its neighbors. This case is clearly seen at the right of Fig. 4 . where the autocorrelation function shows oscillatory characteristics in mixture of $20 \%$ and $50 \%$ Ar. The autocorrelation functions are well converged.

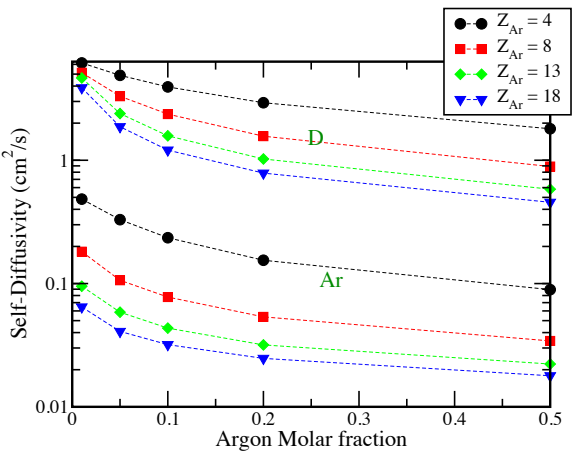

(a) Ion number density is $n=10^{23} / \mathrm{cm}^{3}$

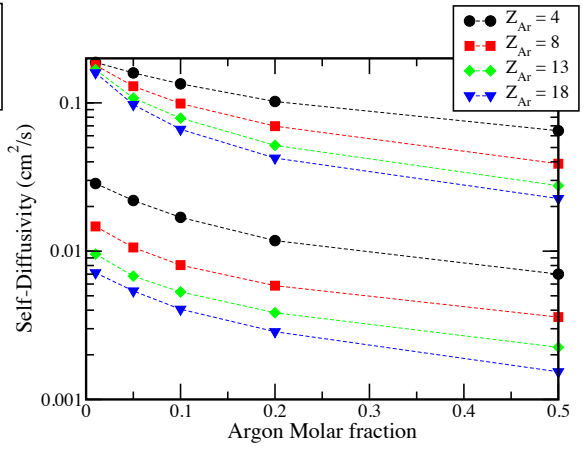

(b) Ion number density is $n=10^{25} / \mathrm{cm}^{3}$

Fig. 5: Self-Diffusivity calculation using molecular dynamics for binary mixtures of deuterium and argon. We have considered mixtures of different Ar mole fraction and different ionization level at a given temperature $T=100 \mathrm{eV}$. Each color will correspond to a given value of Ar ionization. $\mathrm{D}$ is lighter than $\mathrm{Ar}$ and as such its self-diffusivity will have higher value. Therefore in each figure the upper band will correspond to values for D and the lower band to Ar. Each line within these bands correspond to different ionization of Ar.

The full sets of self-diffusivities are shown in Fig. 5 and Tables 1 and 2 In Fig 5 , we have plotted the calculated self-diffusivities as a function of composition and as a function of Ar ionization $Z_{A r}$. The argon mole fraction considered were $X=$ $0.01,0.05,0.1,0.2$ and 0.5 . The value of argon ionizations were $Z_{A r}=4,8,13$ and fully ionized case $Z_{A r}=18$. On the left and the right of Fig. 5 system was kept at ion density $10^{23} / \mathrm{cm}^{3}$ and $10^{25} / \mathrm{cm}^{3}$, respectively. In each plot the self-diffusivity of Ar and $\mathrm{D}$ are plotted together. The values fall in band corresponding to each component, with the upper band corresponding to D. These higher values of D self-diffusion coefficient are related to the fact that its mass is lighter than Ar. In these bands each line corresponds to different Ar ionization $Z_{A r}$. As expected increasing $Z_{A r}$, increases the couplings $\Gamma_{D}, \Gamma_{A}$ of each component, as well as $\Gamma_{\text {eff }}$, which reduces the self-diffusivity of either component. For exactly the same reason if we compare the values plotted in each graph we see a higher value of self-diffusivity for the less dense case with ion density $n=10^{23} / \mathrm{cm}^{3}$ which means lower coupling. In both graphs it is seen that for composition with trace element Ar, the self-diffusivity of $\mathrm{D}$ is almost unaffected from the ionization of Ar. The variance in self-diffusivity values is mainly due to the mass of Ar. The general trend is for the self-diffusivities 
to increase with temperature and to decrease with the density, Ar ionization and Ar mole fraction.

For some compositions we put the above results in Tables 1 and 2 together with the corresponding couplings $-\Gamma_{D}, \Gamma_{A}$ as well as $\Gamma_{e f f}$, dimensionless screening $\kappa$, mass density $\rho$ in $\mathrm{g} / \mathrm{cm}^{3}$. Also as an initial estimate of the Maxwell-Stefan diffusion Eq. (33) we assume that velocity cross-correlation terms are negligible and use the Darken relation (36) given by: $D_{12}^{0}=X D_{D}+(1-X) D_{A r}$. In Tables 1 and 2 this value is input at the last column.

Table 1: Self-diffusion and Darken diffusion values estimated from molecular dynamics for binary mixtures of deuterium and argon. Results correspond to different mixtures at Temperature $100 \mathrm{eV}$ and number density $10^{23} / \mathrm{cm}^{3}$. In the second column $X$ denotes Ar molar fraction.

\begin{tabular}{|c|c|c|c|c|c|c|c|c|c|}
\hline \hline$Z_{A r}$ & $\mathrm{X}$ & $\Gamma_{A r}$ & $\Gamma_{D}$ & $\Gamma_{\text {ff }}$ & $\kappa$ & $\rho\left(\mathrm{g} / \mathrm{cm}^{3}\right)$ & $D_{A r}\left(\mathrm{~cm}^{2} / \mathrm{s}\right)$ & $D_{D}\left(\mathrm{~cm}^{2} / \mathrm{s}\right)$ & $D_{12}^{0}\left(\mathrm{~cm}^{2} / \mathrm{s}\right)$ \\
\hline \multirow{3}{*}{4} & 0.05 & 1.1379 & 0.11289 & 0.16414 & 0.60921 & 0.647444 & 0.32970 & 4.88611 & 0.55752 \\
& 0.1 & 1.18537 & 0.11760 & 0.22438 & 0.64763 & 0.96278 & 0.23533 & 3.93434 & 0.60523 \\
& 0.2 & 1.27032 & 0.12603 & 0.35488 & 0.71826 & 1.59345 & 0.15457 & 2.92675 & 0.70900 \\
\hline \multirow{3}{*}{8} & 0.05 & 3.81095 & 0.11909 & 0.30368 & 0.65993 & 0.647444 & 0.10625 & 3.30412 & 0.26615 \\
& 0.1 & 4.11533 & 0.12860 & 0.52727 & 0.74028 & 0.96278 & 0.07767 & 2.36722 & 0.30663 \\
& 0.2 & 4.61663 & 0.14427 & 1.03874 & 0.87888 & 1.59345 & 0.05368 & 1.56711 & 0.35637 \\
\hline \multirow{3}{*}{13} & 0.05 & 9.05838 & 0.12603 & 0.57264 & 0.71826 & 0.647444 & 0.05858 & 2.39274 & 0.17529 \\
& 0.1 & 10.0728 & 0.14014 & 1.13341 & 0.84167 & 0.96278 & 0.04348 & 1.57843 & 0.19698 \\
& 0.2 & 11.6458 & 0.16203 & 2.45879 & 1.04474 & 1.59345 & 0.031786 & 1.02382 & 0.23019 \\
\hline \multirow{3}{*}{18} & 0.05 & 16.3537 & 0.13228 & 0.94335 & 0.77213 & 0.647444 & 0.04088 & 1.87104 & 0.13239 \\
& 0.1 & 18.5501 & 0.15004 & 1.99006 & 0.93185 & 0.96278 & 0.03194 & 1.20874 & 0.14962 \\
& 0.2 & 21.8295 & 0.17657 & 4.50715 & 1.18681 & 1.59345 & 0.02471 & 0.78650 & 0.17707 \\
\hline \hline
\end{tabular}

At this initial stage it is helpful to compare the Maxwell-Stefan diffusivities with known models. We do this in Fig. 6 for a case with $Z_{A r}=13$ and ion density $10^{25} / \mathrm{cm}^{3}$. Here we compare these results with diffusion from Kinetic Theories [15, 14, 51] based on binary collisions. In this figure the results from one of these theories Paquette et al. [15], that assumes a Yukawa potential when calculating the collision integral, is plotted with solid line. Results from our MD simulations combine in the form of Darken relation are shown with green.

Daligault has also done an extensive MD study for One Component Plasma (OCP) [23] as well as for Yukawa One Component Plasma (YOCP) [68], where he validates the kinetic theories at low coupling and extend it by modeling to moderate coupling. In the strong coupling limit he used an empirical law that describes the diffusion process as ions hopping from a cage to another formed by its neighbors. He extended this aproach for OCP to binary ionic mixtures (BIM) [23] by use of the $\Gamma_{\text {eff }}$ and the mixed plasma frequency as given in Eq. (51). We followed the same scheme to extend the YOCP model to Yukawa mixtures. In Fig. 6results from this model are represented with the long-dashed line. Obviously there is discrepancy between the extension of this model with Darken MD. This is due to the mixed rule that we used to extend this model. 
Table 2: Self-diffusion and Darken diffusion values estimated from molecular dynamics for binary mixtures of deuterium and argon. Results correspond to different mixtures at Temperature $100 \mathrm{eV}$ and number density $10^{25} / \mathrm{cm}^{3}$. In the second column $X$ denotes Ar molar fraction.

\begin{tabular}{|c|c|c|c|c|c|c|c|c|c|}
\hline \hline$Z_{A r}$ & $\mathrm{X}$ & $\Gamma_{A r}$ & $\Gamma_{D}$ & $\Gamma_{\text {eff }}$ & $\kappa$ & $\rho\left(\mathrm{g} / \mathrm{cm}^{3}\right)$ & $D_{A r}\left(\mathrm{~cm}^{2} / \mathrm{s}\right)$ & $D_{D}\left(\mathrm{~cm}^{2} / \mathrm{s}\right)$ & $D_{12}^{0}\left(\mathrm{~cm}^{2} / \mathrm{s}\right)$ \\
\hline & 0.05 & 5.28167 & 0.52400 & 0.761891 & 1.04111 & 64.7444 & 0.02194 & 0.15915 & 0.15228 \\
4 & 0.1 & 5.50199 & 0.54586 & 1.04148 & 1.07903 & 96.278 & 0.01690 & 0.13433 & 0.12258 \\
& 0.2 & 5.89628 & 0.58498 & 1.64724 & 1.14247 & 159.345 & 0.01177 & 0.10212 & 0.08405 \\
\hline & 0.05 & 17.6888 & 0.55277 & 1.40958 & 1.09063 & 64.7444 & 0.01058 & 0.12940 & 0.12346 \\
8 & 0.1 & 19.1017 & 0.59692 & 2.4474 & 1.16079 & 96.278 & 0.00804 & 0.09892 & 0.08983 \\
& 0.2 & 21.4285 & 0.66964 & 4.82142 & 1.26327 & 159.345 & 0.00585 & 0.06961 & 0.05685 \\
\hline \multirow{3}{*}{13} & 0.05 & 42.0453 & 0.58498 & 2.658 & 1.14247 & 64.7444 & 0.00678 & 0.10781 & 0.10275 \\
& 0.1 & 46.7539 & 0.65049 & 5.26084 & 1.23768 & 96.278 & 0.00531 & 0.07889 & 0.07153 \\
& 0.2 & 54.0551 & 0.75208 & 11.4127 & 1.36437 & 159.345 & 0.00384 & 0.05168 & 0.04211 \\
\hline & 0.05 & 75.9072 & 0.61399 & 4.37865 & 1.18619 & 64.7444 & 0.00538 & 0.09728 & 0.09268 \\
18 & 0.1 & 86.1021 & 0.69645 & 9.23702 & 1.29768 & 96.278 & 0.00406 & 0.06630 & 0.06007 \\
& 0.2 & 101.323 & 0.81957 & 20.9204 & 1.43842 & 159.345 & 0.00286 & 0.04241 & 0.03450 \\
\hline \hline
\end{tabular}

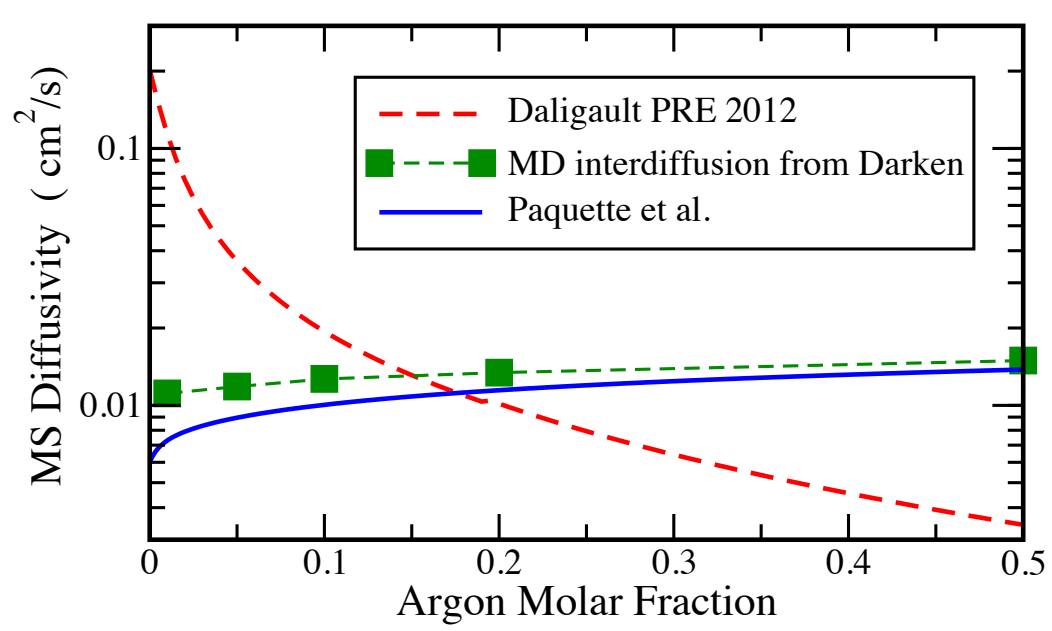

Fig. 6: Maxwell Stefan (MS) diffusivity as a function of Ar mole fraction at a temperature $T=$ $100 \mathrm{eV}$, ion density $10^{25} / \mathrm{cm}^{3}$ and $\mathrm{Ar}$ ionization $Z_{A r}=13$. We compare MS diffusivity from the Darken linear combination 36, with results from kinetic theories from Paquette and Chapman \& Cowling [15] 14]. The long-dashed line represents an extension to mixtures of the Daligault model [68] for YOCP, following his scheme of extending the OCP model to BIM [23], by use of the mixing rule in Eq. (7)

In Fig 6, the agreement with the kinetic theory is fairly good at higher Ar mole fraction, while the discrepancy increases at low Ar mole fraction. This is puzzling, because we would expect the kinetic theories to work very well in the limit of low coupling- low Ar fraction-, where the binary collisions are the dominant factor that drives diffusion. This discrepancy might be due to the fact that we are ignoring the 
cross-correlation terms which might be significant at the low Ar mole fraction. To assess these effects an intense MD study is needed where the Maxwell-Stefan diffusion can be directly computed and then compared against Darken-like relation. We have begun these studies. Also there is a lot of effort on extending kinetic theories to higher coupling [17] through effective potentials, while still retaining the binary collision picture. 


\section{Discussions}

We have reviewed plasma species diffusivity and the various approaches used to calculate it. Kinetic theories, such as the one due to Chapman and Cowling, provide analytic formulas for the diffusivity of weakly coupled mixtures. At stronger coupling the binary collision approximation breaks down and a different technique is needed. MD provides a means of accounting for the many-body effects associated with strong coupling. We have reviewed the self-diffusivity and the MaxwellStefan diffusivity. The statistics of the self-diffusivity calculation are much better than those for the Maxwell-Stefan diffusivity, so we have done some comparisons of the Darken expression for the interdiffusivity with the calculated Maxwell-Stefan diffusivity.

Diffusion is one part of mixing. It would be interesting to apply MD to calculate the hydrodynamic (stirring) part of the mixing as well. MD has been used in molten metals to study the mixing in a shear layer forming the Kelvin-Helmholtz instability, capturing both the vortical flow that sweeps arms of one species into the other and then mixes by diffusion. [69]. Also, it would be interesting to identify regimes in which the diffusion does not follow Fick's law. Non-Fickian diffusion may result from steep gradients, in which the mole fraction changes significantly within one mean free path. There is still much to be learned about diffusion. 


\section{Acknowledgements}

The authors would like to thank Bill Cabot, Kyle Caspersen, John Castor, Jim Glosli, Frank Graziani, Jeff Greenough, Julie Jackson, A. Bruce Langdon, Paul Miller, Michael Murillo, Dave Richards, Mike Surh, Heather Whitley and all the Cimarron team for their contributions and fruitful discussions. One of us T.H. acknowledges enlighting discussion with Jerome Daligault during the IPAM workshop. This work was performed under the auspices of the U.S. Department of Energy by Lawrence Livermore National Laboratory under Contract DE-AC52-07NA27344. This work was funded by the Laboratory Directed Research and Development Program at LLNL under project tracking code 12-SI-005 and 10-ERD-004. We gratefully acknowledge supercomputer time provided through the Institutional Computing Grand Challenge program at LLNL. 


\section{Appendix 1: A general derivation of the Green-Kubo relation for the diffusion of multicomponent mixtures}

In the following we generalize the derivation due to Hansen and McDonald [24] of the Green-Kubo relation of the interdiffusivity of a binary mixture, Eq. (26), to a more general multicomponent mixture. Similar treatment of diffusion in multicomponent mixtures was given by Zhou and Miller [46, 49] in the geophysical context, and by Wheeler and Newman [70] for the electrolytes. The method although cumbersome is, in principle, straightforward.

Let $c_{\alpha}(\mathbf{r}, t)$ denote the mass fraction of component $\alpha$ at position $\mathbf{r}$ and time $t$ in a n-component system. The rate of concentration change is linked to the diffusion flux $\mathbf{J}_{\alpha}(\mathbf{r}, t)$, by the conservation of mass, i.e.,

$$
\rho \frac{\partial c_{\alpha}(\mathbf{r}, t)}{\partial t}+\nabla \cdot \mathbf{J}_{\alpha}(\mathbf{r}, t)=0
$$

where $\rho$ is the mass density of the system. There are $(n-1)$ independent equations from 54. The relation for the barycentric frame $\sum_{\alpha=1}^{n} \mathbf{J}_{\alpha}(\mathbf{r}, t)=0$ completes the system of these equations. In the linear response regime we can relate diffusion fluxes with the gradient of chemical potentials and through it to mass concentration gradient by the following:

$$
\mathbf{J}_{\alpha}(\mathbf{r}, t)=-\rho \sum_{\beta=1}^{n-1} D_{\alpha \beta} \nabla c_{\beta}(\mathbf{r}, t),
$$

where $D_{\alpha \beta}$ are the mutual diffusion coefficients, which form a $(n-1) \times(n-1)$ matrix in an $n$-component system. The following general form of Fick's law for a multicomponent mixture [13] is obtained by combining Eq. [54] with Eq. 55]

$$
\frac{\partial c_{\alpha}(\mathbf{r}, t)}{\partial t}=\sum_{\beta=1}^{n-1} D_{\alpha \beta} \nabla^{2} c_{\beta}(\mathbf{r}, t)
$$

By Fourier transforming in space and Laplace transforming in time the above diffusion equation becomes:

$$
\left|\tilde{c}_{k}(\omega)\right\rangle=\mathscr{D}^{-1}\left|c_{k}\right\rangle
$$

where

$$
\mathscr{D} \equiv-i \omega \mathbf{I}+k^{2} \mathbf{D},
$$

with I the identity matrix. In Eq. $(57\rangle\left|\tilde{c}_{k}(\omega)\right\rangle$ and $\left|c_{k}\right\rangle$ are $n$-1-dimensional vectors in bra-ket notation, whose $\alpha$ th elements are the Fourier-Laplace transform of $c_{\alpha}(\mathbf{r}, t)$ and the Fourier transform of $c_{\alpha}(\mathbf{r}, 0)$, respectively with:

$$
\tilde{c}_{\alpha, k}(\omega)=\int_{0}^{\infty} d t \int d^{3} r c_{\alpha}(\mathbf{r}, t) \exp (-i \mathbf{k} \cdot \mathbf{r}+\omega t),
$$


and

$$
c_{\alpha, k}=\int d^{3} r c_{\alpha}(\mathbf{r}, 0) \exp (-i \mathbf{k} \cdot \mathbf{r}) .
$$

From Eq. 57] we can construct an $(n-1) \times(n-1)$ matrix equation relating different correlation concentration factors:

$$
\mathscr{D}^{-1}=\mathscr{O}^{-1},
$$

where the elements of matrices $\mathscr{O}$ and $\mathscr{C}$ consist of time-dependent and static concentration-correlation functions, respectively, given by:

$$
\mathscr{O}_{\alpha \beta}=\left\langle\tilde{c}_{\alpha, k}(\omega) c_{\beta,-k}\right\rangle
$$

and

$$
\mathscr{C}_{\alpha \beta}=\left\langle c_{\alpha, k} c_{\beta,-k}\right\rangle,
$$

where $\langle\cdots\rangle$ represents statistical ensemble average. Relation 61 can explicitly be written as:

$$
\frac{|\mathscr{D}|_{\beta \alpha}}{|\mathscr{D}|}=\frac{1}{|\mathscr{C}|} \sum_{\gamma=1}^{n-1} \mathscr{O}_{\alpha \gamma}|\mathscr{C}|_{\gamma \beta}
$$

where the elements of the inverse of matrix $\mathscr{D}$ are written as $\left[\mathscr{D}^{-1}\right]_{\beta \alpha}=|\mathscr{D}|_{\alpha \beta} /|\mathscr{D}|$, with $|\mathscr{D}|_{\alpha \beta}$ the cofactor of $\mathscr{D}_{\alpha \beta}$ in the determinant $|\mathscr{D}|$ [38], and similarly for the concentration correlation matrix $\mathscr{C}$.

Taking the inverse Laplace transform of Eq. 64) gives:

$$
-i \omega \frac{|\mathscr{D}|_{\beta \alpha}}{|\mathscr{D}|}-\delta_{\alpha \beta}=\frac{1}{|\mathscr{C}|} \int_{0}^{\infty} \frac{d}{d t}\left(\sum_{\gamma=1}^{n-1}\left\langle c_{\alpha, k}(t) c_{\gamma,-k}\right\rangle|\mathscr{C}|_{\beta \gamma}\right) \exp (i \omega t) \mathrm{d} t
$$

In the long wavelength limit the LHS of Eq. (65) is:

$$
\lim _{k \rightarrow 0}\left(-i \omega \frac{|\mathscr{D}|_{\beta \alpha}}{|\mathscr{D}|}-\delta_{\alpha \beta}\right)=\frac{D_{\alpha \beta} k^{2}}{i \omega}
$$

from which the following expression $D_{\alpha \beta}$ is obtained:

$$
D_{\alpha \beta}=\lim _{\omega \rightarrow 0} \lim _{k \rightarrow 0} \frac{i \omega}{k^{2}|\mathscr{C}|} \int_{0}^{\infty} \frac{d}{d t}\left(\sum_{\gamma=1}^{n-1}\left\langle c_{\alpha, k}(t) c_{\gamma,-k}\right\rangle|\mathscr{C}|_{\beta \gamma}\right) \exp (i \omega t) \mathrm{d} t .
$$

Equation 67) is a generalization of a multicomponent mixture to the one derived by Hansen and McDonald [24]. In the above relation it is essential taking the limit of $k \rightarrow 0$ before $\omega \rightarrow 0$. By use of the mass conservation relation in Fourier space $\rho \frac{\partial c_{\alpha, k}(t)}{\partial t}=-i \mathbf{k} \cdot \mathbf{J}_{\alpha, k}(t)$, in the above equation we can replace the time dependent concentration structure factor by the corresponding diffusion flux correlation func- 
tions. After an integration by parts and use of the time-translational invariance property $\left\langle\mathbf{J}_{1, k}(t) c_{1,-k}\right\rangle=\left\langle\mathbf{J}_{1, k} c_{1,-k}(-t)\right\rangle$, we have:

$$
D_{\alpha \beta}=\lim _{\omega \rightarrow 0} \lim _{k \rightarrow 0} \frac{1}{3 \rho}\left(\sum_{\gamma=1}^{n-1} \frac{|\mathscr{C}|_{\beta \gamma}}{|\mathscr{C}|} \int_{0}^{\infty}\left\langle\mathbf{J}_{\alpha, k}(t) \cdot \mathbf{J}_{\gamma,-k}\right\rangle \exp (i \omega t) \mathrm{d} t\right)
$$

from which we get:

$$
D_{\alpha \beta}=\frac{1}{3 \rho}\left(\sum_{\gamma=1}^{n-1} \frac{\left|\mathscr{C}_{0}\right| \beta \gamma}{\left|\mathscr{C}_{0}\right|} \int_{0}^{\infty}\left\langle\mathbf{J}_{\alpha, k \rightarrow 0}(t) \cdot \mathbf{J}_{\gamma, k \rightarrow 0}\right\rangle \mathrm{d} t\right) .
$$

Here the elements of matrix $\mathscr{C}_{0}$ are given by $\left|\mathscr{C}_{0}\right|_{\alpha \beta} \equiv\left\langle c^{\alpha} c^{\beta}\right\rangle$. This are the long wavelength limit concentration correlation functions. In binary mixtures they are directly related to the concentration structure factor $S_{c c}$ defined by Eq. 27]. Equation 699 is written as a product of two matrices, one whose elements are related to composition-composition structure factor, and the other with elements constructed from Green-Kubo (Maxwell-Stefan) interdiffusion correlation terms. It is straightforward to derive the two component interdiffusion coefficient (26) as a special case of this multicomponent relation 69). In a ternary mixture matrix relation (69) simplifies to:

$$
\left[\begin{array}{ll}
D_{11} & D_{12} \\
D_{21} & D_{22}
\end{array}\right]=\frac{1}{3 \rho\left|C_{0}\right|}\left[\begin{array}{cc}
\left\langle\left(c_{2}\right)^{2}\right\rangle & -\left\langle c_{1} c_{2}\right\rangle \\
-\left\langle c_{1} c_{2}\right\rangle & \left\langle\left(c_{1}\right)^{2}\right\rangle
\end{array}\right] \times\left[\begin{array}{l}
\int_{0}^{\infty}\left\langle\mathbf{J}_{1}(t) \cdot \mathbf{J}_{1}\right\rangle d t \int_{0}^{\infty}\left\langle\mathbf{J}_{1}(t) \cdot \mathbf{J}_{2}\right\rangle d t \\
\int_{0}^{\infty}\left\langle\mathbf{J}_{2}(t) \cdot \mathbf{J}_{1}\right\rangle d t \int_{0}^{\infty}\left\langle\mathbf{J}_{2}(t) \cdot \mathbf{J}_{2}\right\rangle d t
\end{array}\right]
$$

Here

$$
\left|C_{0}\right|=\left\langle\left(c_{1}\right)^{2}\right\rangle\left\langle\left(c_{2}\right)^{2}\right\rangle-\left(\left\langle c_{1} c_{2}\right\rangle\right)^{2}
$$

is the determinant of matrix $\mathbf{C}_{\mathbf{0}}$. In analogy with the binary mixing case we can define a thermodynamic factor matrix $\Phi$ such that:

$$
\left[\begin{array}{ll}
\Phi_{11} & \Phi_{12} \\
\Phi_{21} & \Phi_{22}
\end{array}\right]=\frac{1}{3 \rho\left|C_{0}\right|}\left[\begin{array}{cc}
\left\langle\left(c_{2}\right)^{2}\right\rangle & -\left\langle c_{1} c_{2}\right\rangle \\
-\left\langle c_{1} c_{2}\right\rangle & \left\langle\left(c_{1}\right)^{2}\right\rangle
\end{array}\right]
$$

The Green-Kubo form presented in Eq. (70) provides a simple form that can be used to study ternary mixtures through equilibrium MD. 


\section{Appendix 2: Collision Integrals}

The collision integrals $\Omega_{\alpha \beta}^{(i j)}$ between two particles $\alpha$ and $\beta$ are related to the total cross section after integrating over a Maxwellian velocity distribution. They are given by [14, 15]:

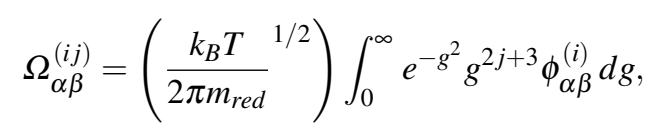

where $g$ is a dimensionless velocity, $m_{r e d} \equiv m_{\alpha} m_{\beta} /\left(m_{\alpha}+m_{\beta}\right)$ is the reduced mass, and $\phi_{\alpha \beta}^{(i)}$ are the collisions cross section for a given energy:

$$
\phi_{\alpha \beta}^{(i)}=2 \pi \int_{0}^{\infty}\left(1-\cos ^{i} \chi_{\alpha \beta}\right) b d b
$$

with the integration over the impact parameter $b$.

In Eq. (74) $\chi_{\alpha \beta}$ is the scattering angle given:

$$
\chi_{\alpha \beta}=\pi-2 \int_{r_{\alpha \beta}^{\min }}^{\infty} \frac{b d r}{r^{2}\left[1-\frac{b^{2}}{r^{2}}-\frac{V_{\alpha \beta}(r)}{g^{2} k_{B} T}\right]^{1 / 2}} .
$$

Here $V_{\alpha \beta}(r)$ is the interaction potential between the particle of type $\alpha$ and $\beta$, and $r_{\alpha \beta}^{\min }$ is the distance of the closest approach between the particle which is the root of the following equation:

$$
1-\frac{b^{2}}{\left(r_{\alpha \beta}^{\min }\right)^{2}}-\frac{V_{\alpha \beta}\left(r_{\alpha \beta}^{\min }\right)}{g^{2} k_{B} T}=0
$$




\section{References}

1. R.E. Rudd, W.H. Cabot, K.J. Caspersen, J.A. Greenough, D.F. Richards, F.H. Streitz, P.L. Miller, Phys. Rev. E 85, 031202 (2012).

2. P. Amendt, O.L. Landen, H.F. Robey, C.K. Li, R.D. Petrasso, Phys. Rev. Lett. 105, 115005 (2010).

3. P. Amendt, S. Wilks, C. Bellei, C.K. Li, R.D. Petrasso, Phys. Plasma 18, 056308 (2011).

4. P. Amendt, C. Bellei, S. Wilks, Phys. Rev. Lett. 109, 075002 (2012).

5. D.B. Dusenbery, Living at Micro Scale (2009).

6. G. Michaud, The Astrophysical Journal 160, 641 (1970).

7. J. Lindl, Phys. Plasmas 2, 3933 (1995).

8. J. Nuckolls, L. Wood, A. Thiessen, G. Zimmerman, Nature 239, 139 (1972).

9. S. Hamaguchi, R.T. Farouki, J. Chem. Phys. 101, 9876 (1994).

10. R.T. Farouki, S. Hamaguchi, J. Chem. Phys. 101, 9885 (1994).

11. S. Hamaguchi, R.T. Farouki, H.E. Dubin, J. Chem. Phys. 105, 7641 (1996).

12. R. Farouki, S. Hamaguchi, Journal of Computational Physics 115(2), 276 (1994).

13. R. Bird, W. Stewart, E. Lightfoot, Transport Phenomena. Wiley International edition (Wiley, 2007).

14. S. Chapman, T. Cowling, The Mathematical Theory of Non-uniform Gases: An Account of the Kinetic Theory of Viscosity, Thermal Conduction and Diffusion in Gases. Cambridge Mathematical Library (Cambridge University Press, 1970).

15. C. Paquette, C. Pelletier, G. Fontaine, G. Michaud, The Astrophysical Journal Supplement Series 61, 177 (1986).

16. H. Ohta, S. Hamaguchi, Physics of Plasmas 7, 4506 (2000).

17. S.D. Baalrud, J. Daligault, Phys. Rev. Lett. 110, 235001 (2013).

18. E.E. Salpeter, Australian. Journal of Physics 7, 373 (1954).

19. J.P. Hansen, G.M. Torrie, P. Vieillefosse, Phys. Rev. A 16, 2153 (1977).

20. S. Bastea, Phys. Rev. E 71, 056405 (2005).

21. Y. Rosenfeld, Phys. Rev. E 47, 2676 (1993).

22. Y. Rosenfeld, E. Nardi, Z. Zinamon, Phys. Rev. Lett. 75, 2490 (1995).

23. J. Daligault, Phys. Rev. Lett. 108, 225004 (2012).

24. J. Hansen, I. McDonald, Theory of Simple Liquids (Elsevier Science, 2006).

25. N. March, M. Tosi, Atomic Dynamics in Liquids. Dover Books on Physics and Chemistry (DOVER PUBN Incorporated, 1991).

26. D. McQuarrie, Statistical Mechanics (Viva Books Private Limited, 2003).

27. J. Horbach, S.K. Das, A. Griesche, M.P. Macht, G. Frohberg, A. Meyer, Phys. Rev. B 75, 174304 (2007).

28. J. Trullàs, J.A. Padró, Phys. Rev. E 50, 1162 (1994).

29. J. Daligault, Phys. Rev. Lett. 96, 065003 (2006).

30. A. Einstein, Annalen der Physik 322(8), 549 (1905).

31. R. Kubo, Journal of the Physical Society of Japan 12(6), 570 (1957).

32. R. Kubo, M. Yokota, S. Nakajima, Journal of the Physical Society of Japan 12(11), 1203 (1957).

33. R. Zwanzig, J. Chem. Phys. 40, 2527 (1964).

34. R. Zwanzig, Annual Review of Physical Chemistry 16, 67 (1965).

35. D.B. Boercker, E.L. Pollock, Phys. Rev. A 36, 1779 (1987).

36. C. Cohen, J.W.H. Sutherland, J.M. Deutch, Phys. Chem. Liq. 2, 213 (1971).

37. A.B. Bhatia, D.E. Thornton, Phys. Rev. B 2, 3004 (1970)

38. J.G. Kirkwood, F.P. Buff, J. Chem. Phys. 19, 774 (1951).

39. J.P. Hansen, G.M. Torrie, P. Vieillefosse, Phys. Rev. A 16, 2153 (1977).

40. D. Léger, C. Deutsch, Phys. Rev. A 37, 4916 (1988).

41. J. Stefan, Akad. Wiss. Wien Abt. II, 63 (1871).

42. J.P. Hansen, F. Joly, I.R. McDonald, Physica 132A, 4722 (1985).

43. M. Schoen, C. Hoheisel, Mol. Phys. 52, 1029 (1984). 
44. D.L. Jolly, R.J. Bearman, Mol. Phys. 41, 137 (1980).

45. D.W. McCall, D.C. Douglass, J. Phys. Chem. 71, 987 (1967)

46. Y. Zhou, G.H. Miller, Phys. Rev. E 53, 1587 (1996).

47. L.S. Darken, Trans. AIME 175, 184 (1948).

48. D.C. Douglass, H.L. Frisch, J. Phys. Chem. 73, 3039 (1969).

49. Y. Zhou, G.H. Miller, J. Phys. Chem. 100, 5516 (1996).

50. J.P. Hansen, I.R. McDonald, Phys. Rev. A 11, 2111 (1975).

51. L. Spitzer, Physics of fully ionized gases. Interscience tracts on physics and astronomy (Interscience Publishers, 1962).

52. D. Frenkel, B. Smit, Understanding Molecular Simulation: From Algorithms to Applications. Computational science (Elsevier Science, 2001).

53. G. Ciccotti, D. Frenkel, I. McDonald, Simulation of liquids and solids: molecular dynamics and Monte Carlo methods in statistical mechanics. North-Holland personal library (NorthHolland, 1987).

54. N. Ashcroft, N. Mermin, Solid state physics. Science: Physics (Saunders College, 1976).

55. B. Firey, N.W. Ashcroft, Phys. Rev. A 15, 2072 (1977).

56. P. Debye, E. Hückel, Phys. Z 24, 185 (1923).

57. D. Pines, P. Nozieres, The Theory of Quantum Fluids(Addison-Wesley, Redwood, CA, 1990) (1990).

58. S. Itchimaru, Statistical Plasma Physics(Addison-Wesley, Reading, MA, 1991) (1991).

59. G. Gregori, S.H. Glenzer, W. Rozmus, R.W. Lee, O.L. Landen, Phys. Rev. E 67, 026412 (2003).

60. M.S. Murillo, High Energy Density Physics 4(12), 49 (2008).

61. P.P. Ewald, Ann. Phys. 64, 253 (1921).

62. M. Desrno, C. Holm, J. Chem. Phys. 109, 7678 (1998).

63. E.L. Pollock, J. Glosli, Comp. Phys. Comm. 95, 93 (1996).

64. S. Nosé, J. Chem. Phys. 81, 511 (1984).

65. W.G. Hoover, Phys. Rev. A 31, 1695 (1985).

66. R.P. Feynman, N. Metropolis, E. Teller, Phys. Rev. 75, 1561 (1949).

67. M. Vergeles, G. Szamel, J. Chem. Phys. 110, 3009 (1999).

68. J. Daligault, Phys. Rev. E 86, 047401 (2012).

69. J. Glosli, K. Caspersen, D. Richards, R. Rudd, F. Streitz, J. Gunnels, in Proc. Supercomputing 2007 (SC07), Reno, NV, Nov. 2007. UCRL-CONF-230679 pp. 276 - 287 (2007).

70. D.R. Wheeler, J. Newman, J. Phys. Chem. B 108, 18353 (2004). 